\title{
Dynamique de reconstitution de la biodiversité végétale de la forêt classée de Foumbou (Nord de la Côte d'Ivoire)
}

\author{
Adama TAONDA $^{1 *}$, Anny Estelle N'GUESSAN ${ }^{2}$ et Justin N'DJA KASSI ${ }^{2}$ \\ ${ }^{1}$ Centre d'Excellence Africain sur le Changement Climatique, la Biodiversité et l'Agriculture Durable (CEA- \\ CCBAD) de l'Université Félix Houphouët-Boigny, UFR Biosciences, Laboratoire de Botanique, Côte d'Ivoire, \\ 22 BP 582 Abidjan 22, Côte d'Ivoire. \\ ${ }^{2}$ Université Félix Houphouët-Boigny, UFR Biosciences, Laboratoire de Botanique, Côte d'Ivoire, 22 BP 582 \\ Abidjan 22, Côte d'Ivoire. \\ *Auteur correspondant ; E-mail : adamataonda1 @ gmail.com
}

Received: 10-06-2021

Accepted: $20-11-2021$

Published: 31-12-2021

\section{RESUME}

La forêt est un milieu essentiellement dynamique dont les multiples composants sont en perpétuelle évolution. La forêt classée de Foumbou, situé dans le département de Korhogo au Nord de la Côte d'Ivoire, est confrontée à l'orpaillage clandestin couplé aux infiltrations paysannes. Ces exploitations demeurent une préoccupation écologique majeure car elles menacent les services écosystémiques. Ces travaux avaient pour objectif d'évaluer la diversité floristique de la forêt classée de Foumbou dans une perspective de gestion durable forestière de la biodiversité. Les méthodes de relevé de surfaces associées à des inventaires itinérants ont été adoptées lors de ces travaux dans vingt parcelles de $2000 \mathrm{~m}^{2}$ chacune. Les analyses multivariées ont permis d'identifier cinq stades de succession secondaire postculturale le long d'une chronoséquence de 35 ans. Les paramètres de composition augmentent avec l'âge de la jachère (biotopes). La forêt renferme, au total, 259 espèces, réparties en 191 genres et 56 familles. Du point de vue de la composition floristique, cette forêt est riche en Fabaceae, en Rubiacea, en Combretaceae, en Malvaceae, en Moraceae, en Poaceae et en Lamiaceae. Dans cette étude, les biotopes les plus diversifiés sont les jachères de plus de 20 ans et les Forêts anciennes. Le site renferme également des espèces endémiques et des espèces à statuts particuliers. La flore de cette forêt est diversifiée avec une répartition équitable des espèces au sein des biotopes. De ce fait, des actions de gestion intégré et participative de cette forêt s'imposent pour minimiser sa dégradation.

() 2021 International Formulae Group. All rights reserved.

Mots clés : Dynamique de reconstitution, biodiversité végétale, forêt classée de Foumbou.

\section{Dynamics of reconstitution of the vegetal biodiversity of the Foumbou class forest (north of the Ivory Coast)}

\begin{abstract}
The forest is an essentially dynamic environment whose multiple components are in perpetual evolution. The classified forest of Foumbou is confronted with clandestine gold panning coupled with peasant infiltration. These operations remain a major ecological concern because they threaten ecosystem services. The objective of this work was to assess the floristic diversity of the floristic diversity of the classified forest of Foumbou in order
\end{abstract}


to promote its sustainable management. Surface survey methods and itinerant inventories were adopted during this work in twenty plots, sixteen of which were in post-cultivation fallows. Multivariate analyses identified five stages of postcultural secondary succession along a 35-year chronosequence. The compositional parameters increase with the age of fallow land. The forest contains a total of 259 species, divided into 191 genera and 56 families. From the point of view of floristic composition, this forest is rich in Fabaceae, Rubiacea, Combretaceae, Malvaceae, Moraceae, Poaceae and Lamiaceae. Of this study, the most diversified are the fallow land of more than 20 years and the Old Forest. The flora of the estate is diversified with an equitable distribution of species within the biotopes. Integrated and participative management of the forest is necessary to minimise the degradation of the site.

(C) 2021 International Formulae Group. All rights reserved.

Keywords: Dynamics of reconstitution, plant biodiversity, floristic diversity, classified forest of Foumbou.

\section{INTRODUCTION}

La forêt est un milieu essentiellement dynamique dont les multiples composants sont en perpétuelle évolution (N'Guessan et al., 2019; Rarivoson, 2019). En effet, les écosystèmes forestiers jouent un rôle crucial dans le bien-être des populations humaines (Akossongo, 2004). Ils rendent de nombreux services qui sont entre autres : la régulation du climat, le recyclage des nutriments, la stabilisation des sols, la création d'habitats naturels, sans oublier toute une pléiade de loisirs en plein air (Fearnside, 2005 ; Locatelli, 2011). Ils fournissent également des biens tels que : nourritures, médicaments et un large éventail des produits forestiers. Lorsque ces écosystèmes sont bien gérés, ils constituent des puits de carbone et des réservoirs de la biodiversité les plus importants de la planète (Roxane, 2015 ; Marion, 2018). Aussi, ces écosystèmes jouent un rôle fondamental dans la lutte contre la pauvreté en milieu rural en assurant la sécurité alimentaire et en fournissant les moyens de subsistance (FAO, 2015). Dans ce cadre, ils fournissent d'importante quantité de ressources naturelles pour la plupart des pays tropicaux et jouent un rôle important dans la conservation de la biodiversité et l'équilibre écologique (N'guessan et al., 2019). Malheureusement, les forêts du monde continuent de se rétrécir. En effet, avec l'accroissement démographique, davantage de terres forestières sont convertis en terres agricoles ou à d'autres utilisations (FAO, 2020). Pour Kouadio et al. (2018), leurs caractéristiques structurales et leurs dynamiques floristiques sont constamment modifiées par diverses activités telles le changement d'utilisation des terres pour l'intensification de l'agriculture. En outre, la perte de ces ressources provoque un affaiblissement et de la capacité des communautés tributaires des forêts à en tirer des revenus, de la nourriture et des médicaments (Cissé et al., 2020). Dans ce contexte, les peuplements de nombreuses espèces dans ces écosystèmes se trouvent dans une dynamique régressive. Les évaluations faites par la FAO montrent que l'Afrique a un taux de déforestation annuel de 3,9 millions d'hectares (FAO, 2020). En plus, les détenteurs de droit coutumier sur les terres forestières mènent à grand échelle des activités de conversion des forêts rurales en espaces agricoles (Toko et al., 2012). Cette pratique met en danger la survie de plusieurs espèces animales et végétales dans les différentes régions (Kouadio et al., 2018). La conversion de ces écosystèmes en terre agricole accentue la concentration des gaz à effet de serre dans l'atmosphère, ce qui intensifie le réchauffement climatique (Saïdou et al., 2012). Ce qui a pour conséquence le changement climatique. La modification de ces paramètres climatiques peut influencer la croissance des arbres et la fréquence de l'intensité des incendies ainsi que l'incidence des ravageurs qui accentuent des dommages causés aux forêts par des conditions météorologiques extrêmes telles que la sécheresse, les inondations et les tempêtes (FAO, 2002 ; Martel et Picard, 2013). De nos jours, le changement climatique est l'un des principaux problèmes environnementaux auquel l'humanité est confrontée. En Afrique, 
et particulièrement En Afrique de l'Ouest, et ce surtout dans la zone tropicale, on constate de manière générale la régression continue des massifs forestiers (Toko et al., 2012). Ce qui a pour conséquence la régression des écosystèmes forestiers.

En Côte d'Ivoire, les forêts claires sont situées dans la partie Nord. Ces forêts jouent un rôle essentiel dans l'atténuation du changement climatique (Sanogo, 2020). Avec la perte du couvert forestier ivoirien, nous nous interrogeons à l'instar de la forêt classée de Badénou (Gboze et al., 2020), l'état de vulnérabilité de la forêt classée de Foumbou, située dans la Région de Korhogo au nord de la Côte d'Ivoire qui est cependant confrontée à l'orpaillage clandestin couplé aux infiltrations paysannes. Ces exploitations demeurent une préoccupation écologique majeure car elles menacent les services écosystémiques et contribuent ainsi à l'émission des GES. À l'instar de la forêt classée de Badénou (Gbozé et al., 2020), la forêt classée de Foumbou, estelle susceptible d'être plus vulnérable aux effets de changements climatiques vu son état ? Aussi, bien que, les caractéristiques taxonomiques de cette réplique forestière constituent un enjeu majeur puisqu'elle offre des services écosystémiques qui contribuent à la réduction de la vulnérabilité de la population, les connaissances scientifiques sur sa composition floristique restent toujours peu connues. Cette étude a été entreprise pour pallier à la rareté des données chiffrées sur la flore et la dynamique de reconstitution posculturale de cette forêt. L'objectif principal de cette étude était d'évaluer la diversité floristique de cette formation forestière qui est en perpétuel dégradation. Spécifiquement, elle vise à déterminer la richesse floristique de cette forêt classée et ensuite la dynamique de succession des espèces ligneuses.

\section{MATÉRIEL ET MÉTHODES Aire d'étude}

La forêt classée de Foumbou est située au Nord de la Côte d'Ivoire, précisément dans les Départements de Korhogo, de Dianra et de Dikodougou. La forêt est donc située dans les régions administratives du Poro et du Béré.
Elle est délimitée à l'Est par les coordonnées géographiques $\left(08^{\circ} 57^{\prime} 27^{\prime \prime} ; 05^{\circ} 53^{\prime} 04^{\prime \prime}\right)$ à l'Ouest par (08 $\left.48^{\circ} 25^{\prime \prime} ; 06^{\circ} 11^{\prime} 08^{\prime \prime}\right)$, au Sud par $\left(08^{\circ} 48^{\prime} 53^{\prime \prime}\right.$; $\left.06^{\circ} 03^{\prime} 37^{\prime \prime}\right)$ ) et au Nord par $\left(09^{\circ} 05^{\prime} 15^{\prime \prime} ; 06^{\circ} 00^{\prime} 21^{\prime \prime}\right)$. C'est un milieu où le relief est dominé par le plateau, avec un réseau hydrographique caractérisé par un affluent du Bandama (Badénou). Le réseau hydrographique comprend des cours d'eau permanents et des cours d'eau temporaires (Kassé, 2009). La forêt classée de Foumbou est située dans une zone dominée par le climat soudano-guinéen. Elle est caractérisée par deux saisons (sèche et pluvieuse). Le climat est conditionné par l'influence alternée de deux courants atmosphériques. L'un de ces courants est l'harmattan qui souffle du Nord-Est pendant une partie importante du mois de décembre à mars. L'autre courant atmosphérique dénommé improprement "mousson" est originaire du Sud-Ouest et apporte des masses d'air océaniques humides. Il prédomine d'une façon presqu'exclusive de la fin du mois de mars à la fin du mois de novembre.

\section{Méthode}

Le plan d'échantillonnage a été conçu de manière à placer des parcelles dans les différents types de végétations rencontrées sur le site à savoir: les forêts anciennes, les jachères post-culturales classées en quatre (4) groupements de végétaux en fonction de l'âge tout en tenant compte du type de sol (sol hydromorphe et feralitique). Ce sont: les jachères de 1-10 ans (catégorie 1), les jachères de 11-19 ans (catégorie 2), les jachères de 2029 ans (catégorie 3 ) et les jachères de 30 ans et plus (catégorie 4) et les forêts anciennes. Au total, 20 parcelles ont été installées. La taille des échantillons était de $100 \mathrm{~m}$ x $20 \mathrm{~m} \mathrm{(2000}$ $\mathrm{m}^{2}$ ). Les relevés de végétation consistaient à recenser toutes les espèces vasculaires présentes dans la surface-échantillon (2000 $\mathrm{m}^{2}$ ), accompagnées de leur coefficient d'abondance-dominance selon l'échelle de Kassi et al. (2011). Les coefficients d'abondance-dominance ont par la suite été convertis conformément aux recommandations de Kassi (2006) pour les analyses numériques. 
Des inventaires itinérants complémentaires ont été menés dans toutes les parties de la forêt y compris les cultures. Ces inventaires ont permis de compléter la liste floristique. L'identification de ces espèces botaniques inventoriées a été faite sur le terrain grâce à la clé d'identification de Hawthorne (1996) et aux travaux de Aké-Assi (1984). Les noms des espèces inventoriées ont été mises à jour à partir de Lebrun et Stork (1991-1997). La nomenclature adoptée pour les familles est celle d'APG IV (2016).

\section{Analyse des données floristique}

Un catalogue floristique reprenant toutes les espèces présentes dans les vingt (20) relevés de végétations, a été établi, auxquelles les espèces supplémentaires répertoriées au cours de la l'inventaire itinérante ont été complétées. Pour chaque espèce identifiée, le type biologique, la phytochorie et le mode de dissémination ont été établie. Les ouvrages botaniques (White, 1983 ; Lebrun et Stork, 1991-1997; Aké-Assi, 2001-2002) ont servir de référence. La richesse floristique d'un territoire est le nombre, d'espèces recensées à l'intérieur de ses limites. En vue d'évaluer cette richesse floristique, une base de données floristique contenant les données taxonomiques (Famille, genre, espèce, sousespèce ou variété et nom de l'auteur) a été réalisée. Pour les espèces identifiées, les traits biologiques retenus ont été définis de la manière suivante : les types biologiques liés à l'état phénologique de l'espèce rencontrée ont été utilisés. Cela a permis de distinguer les hémicryptophytes $(\mathrm{H})$, les chaméphytes $(\mathrm{Ch})$, les géophytes $(\mathrm{G})$, les épiphytes (Ep), et les phanérophytes $(\mathrm{Ph})$. En fonction de la hauteur de ces axes aériens, les phanérophytes sont subdivisées en mégaphanérophytes (MP: arbres de plus de $30 \mathrm{~m}$ de haut), mésophanérophytes (Mp: arbres de 8 à $30 \mathrm{~m}$ de haut), microphanérophytes (mp: arbustes de 2 à $8 \mathrm{~m}$ de haut) et nanophanérophytes (np: sousarbuste moins de $2 \mathrm{~m}$ de haut).

Selon Aké-Assi (1984), la zoochorie est le mode de dissémination par les animaux. Il peut être externe (épizoochorie) ou interne (endozoochorie). $\mathrm{La}$ barochorie, l'anémochores et les phytochories dans les travaux de Aké-Assi (1984), distinguent les espèces disséminées sous l'effet de la pesanteur, par le vent et les espèces forestières qui se rencontrent naturellement dans la région phytogéographique Guinéo-Congolaise (GC), les espèces savanicoles dans la région phytogéographique Soudano-Zambézienne (SZ), les espèces de transition forêt-savanes (GC-SZ) et les espèces introduites (I). Cet auteur ajoute à ces types chorologiques, ceux proposés par White (1983) tel que les espèces réparties dans toutes les régions tropicales du globe (Pan : pantropicales); espèces présentes aussi bien en Afrique tropicale, en Asie tropicale, Australie et à Madagascar (Pal : paléotropicales); espèces présentes en Afrique et en Amérique tropicale (Aam : afroaméricaines); espèces rencontrées dans toute l'Afrique tropicale (AT : afro-tropicales) ; espèces plurirégionales africaines (PA). La caractérisation de ces paramètres floristiques a permis d'apprécier le type d'espèce présent dans la forêt et leur mode de régénération et de conservation. En vue d'une analyse exploratoire multidimensionnelle, les données provenant des 20 relevés de végétations sont enregistrées sous la forme d'une matrice contenant les espèces en ligne, les relevés en colonne et les coefficients d'abondancedominance aux intersections. Cette matrice de données a été utilisée pour les analyses de détermination des groupements végétaux à partir de la classification des relevés (CHA) et de l'ordination (DCA) en utilisant la méthode Ward les distances Euclidiennes (McCune et Grace, 2002). Les espèces indicatrices ou espèces ayant les plus grands scores (les plus fréquentes) pour chaque groupe de végétation issu de la CHA et de la DCA ont été utilisées pour caractériser ces groupements végétaux.

\section{La diversité quantitative de la flore}

Les méthodes univariées (calcul des indices) ont été utilisées pour la caractérisation des groupements identifiés. Plusieurs indices permettent d'apprécier cette diversité.

\section{L'indice de Shannon (1948)}

L'indice de Shannon (H') mesure la composition en espèce d'un peuplement en 
tenant compte de la richesse spécifique et de l'abondance relative. Il a une valeur comprise normalement entre 0 et 5 selon Magurran (2004), lorsqu'il est calculé avec le Logarithme népérien (ln). Si nous désignons par N l'effectif total des $\mathrm{S}$ espèces considérées, ni l'effectif des individus d'une espèce $\mathrm{i}$ et $\ln (\mathrm{ni} / \mathrm{N})$ l'abondance relative de l'espèce i. Il se calcule en utilisant la formule suivante : $\quad H^{\prime}=-\Sigma$ $[(\mathrm{ni} / \mathrm{N}) \mathrm{x} \ln (\mathrm{ni} / \mathrm{N})]$

Cet indice varie de 0 (une seule espèce présente) à $\ln (\mathrm{S})$ (toutes les espèces présentes ont une même abondance, donc bonne diversité).

\section{Indice d'équitabilité de Piélou (1966)}

Pour un peuplement, l'équitabilité nous renseigne sur la répartition des effectifs entre les différentes espèces. Le calcul de l'indice de diversité spécifique s'accompagne toujours de celui de l'équitabilité, car deux peuplements à physionomie différente, peuvent avoir la même diversité (Kouadio et al., 2018). L'indice d'équitabilité (E) de Piélou (1966), appelé également indice d'équirépartition (Blondel, 1979) est le rapport entre l'indice de Shannon de l'échantillon et la diversité maximale. L'équitabilité varie de 0 à 1 . Elle tend vers 0 quand la quasi-totalité des effectifs est concentrée sur une espèce et vers 1 lorsque toutes les espèces ont la même abondance. Dans le cas où cet indice tend vers 1 , le milieu en question est dit équilibré.

Il se calcule selon la formule mathématique suivante :

$$
\mathrm{E}=\mathrm{H}^{\prime} / \ln \mathrm{S}
$$

Dans cette formule, E représente l'indice d'équitabilité de Piélou ; H', l'indice de Shannon et $\ln (S)$ représente la diversité maximale du biotope avec $\mathrm{S}$ le nombre total d'espèces du biotope donné.

\section{Coefficient de similitude}

$\mathrm{La}$ ressemblance floristique entre les différents biotopes du site en vue d'évaluer la diversité $\beta$ sera analysée à travers le coefficient de similitude de Sorensen. Si nous désignons par A et B les nombres respectifs d'espèces de deux listes appartenant à 2 biotopes I et II et par $\mathrm{C}$, le nombre d'espèces communes aux deux listes, le coefficient de similitude Ks est donné par la formule suivante :

$$
\mathrm{Ks}=2 \mathrm{C} \times 100 /(\mathrm{A}+\mathrm{B})
$$

Cet indice varie de 0 à 100 . Plus les deux listes floristiques ont des espèces en commun, plus Ks tend vers 100. Plus les deux listes floristiques sont différentes, plus Ks tend vers 0 .

\section{RÉSULTATS}

\section{Richesse floristique}

Des relevés de surfaces complétés par des relevés itinérants ont permis d'inventorier 259 espèces reparties en 191 genres et 56 familles. La famille la plus diversifiée est celle des Fabaceae avec 50 espèces soit un taux de $19,31 \%$. Elle est suivie des Rubiaceae (17 espèces soit $6,56 \%$ ), des Combretaceae, des Malvaceae, des Moraceae (avec chacune 14 espèces soit $5,41 \%$ ); des Poaceae, des Lamiaceae avec respectivement $12(4,63 \%)$ et $11(4.25 \%)$ espèces chacune. Certaines familles comme les Verbenaceae, Salicaceae, Polygalaceae, Myrtraceae, etc., sont moins représentées avec une seule espèce soit un taux de $0,39 \%$. Parmi les espèces du sous-bois, il existe les jeunes arbres en pleine croissance et des arbustes comme: Pericopsis laxiflora (Benth) Meeuv. (Fabaceae), Detarium microcarpum Guill. \& Perr. (Fabaceae), Cassia sieberiana DC. (Facaceae), Diospiros mespiliformis A. Rich. (Ebenaceae), généralement en touffe. Les herbacées sont plus abondantes dans les jachères, notamment dans les jeunes jachères allant de 1 à 10 ans essentiellement recouvertes Hyptis suavolensis (L.) Poit. (Lamiaceae). Certaines espèces n'ont été inventoriées que dans une (01) parcelle sur les 20 : Securidaca longependunculata Fresen (Polygalaceae), dans deux (02) parcelles : Sterculia setigera Delile (Malvaceae). La Figure 1 présente les espèces les plus représentatives dans la forêt. Ces travaux montrent que la richesse spécifique augmente avec l'âge de la jachère, passant d'une moyenne de 31 espèces dans les plus jeunes stades (1-10 ans) à 97 espèces dans les jachères les plus âgées (30 ans et plus). Dans les vingt parcelles échantillonnées, 158 espèces qui ont 
un diamètre $\geq$ à $2,5 \mathrm{~cm}$. Les familles dominantes dans ces forêts anciennes sont celles des Fabaceae (24 espèces), des Combretaceae (8 espèces), des Rubiaceae et des Malvaceae avec 7 espèces chacune et les autres familles qui sont faiblement représentées. Les jachères de 30 ans et plus, renferment 27 familles avec 57 genres $(74,03 \%$ de l'effectif total) reparties en $74(75,51 \%)$ des espèces totales. Celles-ci sont dominées également par les Fabaceae (20 espèces), les Combretaceae (10 espèces), les Rubiaceae ( 8 espèces Phyllantaceae et les Lamiaceae 7 espèces chacune. Au niveau des jachères de 2130 ans, soixante-deux (62) espèces ont été inventoriées. Celles-ci se répartissent en 56 genres et 25 familles. La famille des Fabaceae dominent avec 16 espèces suivent celles des Malvaceae (8 espèces). Les jachères de 11-20 ans renferment 51 espèces reparties entre 50 genres et 22 familles. Ces familles sont également dominées par les Fabaceae (14 espèces), suivent les Moraceae et les Combretaceae (6 espèces) chacune. Les jeunes jachères renferment en moyenne 31 espèces repartis en 29 genres et 16 familles chacune. Avec une dominance de la famille des Fabaceae (9 espèces), suivies par les Asteraceae (6 espèces) et les Lamiaceae (3 espèces) etc. Le spectre suivant montre la répartition des genres en fonction des familles. Les autres familles ayant un seul genre sont regroupées en autres.

\section{Types biologiques}

Les espèces recensées dans la forêt classée de Foumbou appartiennent à 9 grands types biologiques. Le spectre des types biologiques des espèces (Figure 2), inventoriées met en évidences une nette dominance des microphanérophytes (59\%), viennent ensuite les nanophanérophytes (16\%), les mésophanérophytes (6\%), les thérophytes avec 12 espèces soit $4 \%$ des espèces. Les Géophytes et les hydrophytes présentent chacune 10 espèces soit $4 \%$; les chaméphytes représentent 7 espèces soit $4 \%$ des espèces inventoriées, et les autres types sont représentés par une espèce chacune.
Affinités

\section{(phytogéographique)}

chorologiques

La flore de la forêt classée de Foumbou est constituée majoritairement d'espèces de transition ou de liaison guinéo-congolaisessoudano-zambéziennes (GC-SZ), avec 104 espèces soit un taux de 40\% (Figure 3). Les espèces de la zone soudano-zambésiennes (SZ) et de la zone guinéo-congolaises (GC) suivent respectivement avec $67(26 \%)$ et 47 (17\%) espèces. Les espèces afro-tropicales (AT), suivent avec 40 espèces (soit $4 \%$ de l'effectif total). La répartition des autres espèces inventoriées selon la classification de White se présente comme suite : pantropicales (Pan, 11 espèces, soit 4\%); espèces plurirégionales africaines (AT et PA) respectivement 10 et 9 espèces chacune, soit $3,16 \%$ ), les espèces guinéeo-congolaises endémiques Ouest-africaines (GCW) sont aux nombres de 4 soit un taux de $2 \%$ des espèces total. Les indo-Maléennes (Ind-M, 4 espèces). Les autres sont constituées des afroaméricaines (Aam, 02 espèces, soit $0,77 \%$, Ind et Neo une espèce chacune, soit $0,39 \%$ ).

\section{Mode de dissémination des diaspores}

Les espèces endozoochores et anémochores (Figure 4), avec respectivement 185 et 112 espèces chacune soit $46 \%$ et $43 \%$ de l'effectif total, sont les modes de disséminations le plus représentatif de la forêt. On enregistre également la présence des espèces épizoochores et barochores qui sont les moins présentées avec une proportion respective de 5\% (13 espèces) et $6 \%$ (16 espèces). Dans les différents biotopes, il y'a une dominance endozoochores et anémochores.

\section{Richesse qualitative : les espèces à statut particulier}

La comparaison des espèces recensées à celles de l'UICN et de Aké-Assi a permis d'identifier respectivement 55 espèces soit une proportion de $(21,23 \%)$ et 2 espèces (soit $0,77 \%$ ) appartement à ces deux listes. Celles figurant sur la liste de Aké-Assi, sont Detarium microcarpum et Syzygium guineense. Toutes les espèces de la liste de l'UICN sont de la 
catégorie préoccupation mineur. Parmi les espèces recensées dans la forêt, 2 (soit un taux de $0,77 \%$ de l'effectif total) appartement à ces deux listes. Celles sont endémiques Ouest Africaine (GCW). L'analyse révèle que ce site comporte 7 espèces de bois d'œuvres dont trois (3) de la catégorie P1 et quatre (4) de la catégorie P3 (Tableau 1). Parmi les espèces recensées dans la forêt classée de Foumbou, huit espèces sont classées à statuts particulier réparties comme suit: deux espèces endémiques Ouest africaine (Amorphophallus accrensi N.E.Br., Bulbophyllum calyptratum var. lucifugum (Sum.) Vern.), trois espèces mineurs (Commelina benghalensis L., Desmodium adscendens (Sw.) DC. var. adscendens, Desmodium salicifolium (Poir.) DC.), une espèce à risque faible et classée dans la liste de Aké-Assi (Milicia excelsa (Poir.) DC.), une espèce vulnérable (Albizia ferruginea (Guill. \& Perr.) Benth.).

\section{Indices de Diversité floristique Indice de diversité spécifique}

Deux indices ont été calculés à partir des parcelles disposées dans les différentes formations végétales parcourues. $\mathrm{Ce}$ sont l'indice de Shannon et l'indice d'équitabilité de Piélou (Tableau 2). L'indice de diversité de Shannon varie de 2,32 pour les plus jeunes jachères à 3,29 pour les jachères de 30 ans et plus et 3,04 pour les jachères de 21-30 ans et 11-20 ans. Par ailleurs, ces valeurs restent néanmoins inférieures à celles observées dans les forêts anciennes $(3,31)$. Les milieux dont la valeur de l'indice est élevée bénéficient des conditions écologiques et de stabilité relativement favorables au maintien de plusieurs groupes d'espèces que dans les milieux à faible valeur. L'indice d'équitabilité tend vers 1 sur l'ensemble des biotopes. Il varie de 0,68 pour les jachères $\leq 10$ ans à 0,72 pour les forêts anciennes. Les jachères $\geq 30$ ans ont pour indices 0,78 . Les jachères de 11 à 20 et $20-30$ ans ont 0,77 . Ces valeurs révèlent que la répartition des espèces se fait de manière régulière et équitable qu'il n'existe pas de dominance d'une espèce au sein des biotopes.

\section{Ressemblance floristique}

Le coefficient de similitude de Sorensen calculé (Tableau 3) entre les différents biotopes montrent qu'il y a une forte ressemblance floristique entre les jachères matures (30 ans et plus) et les forêts anciennes $(0,79)$. Tout comme entre les jachères de 21 à 30 ans et les forêts anciennes. Elle est faible entre les jeunes jachères (1-10 ans) et les autres jachères $(\approx 60 \%)$.

\section{Détermination des groupements des végétaux}

Au seuil de $75 \%$ d'informations (Figure 5), la CHA permet de dégager cinq types de formation végétale. Dans le détail, la première branche du cluster, permet de dégager deux grands groupes: les forêts anciennes d'une part, et d'autre part, les jachères postculturales. La deuxième branche du cluster permet quant à elle de séparer les plus jeunes jachères (du groupe I) des autres groupes de jachères. L'âge est donc le principal facteur discriminant. Il permet d'identifier cinq groupes. Ces groupes coïncident globalement avec les quatre classes d'âge des jachères (de I à IV) et la forêt ancienne (Groupe V) avec des espèces caractéristiques de chaque milieu. Le groupe I est constitué de jachères récentes (1 à 10 ans). Le groupe II est celui des jachères de 11 à 20 ans, les groupes III et IV sont constitués respectivement des jachères de 20 à 29 ans et des jachères de 30 ans et plus. Les jeunes jachères sont caractérisées par l'abondance de certaines espèces savanicoles. Il s'agit de Parkia biglobosa (Jacq.) Benth. Le groupe II (jachères 11-20 ans) est constitué majoritairement des espèces savanicoles qui sont, entre autre, Pterocarpus erinaceus et Afzelia africana. Les groupes III et IV (20 à 29 ans et des jachères de 30 ans et plus) sont constitués de Terminalia macroptera Guill. \& Perr. et Margaritaria discö̈dea (Baill.) Müll. Arg., quant aux forêts anciennes, les espèces indicatrices sont entre autres Kaya senegalens et Lophira lanceolata van Tiegh. ex Keay (Figure 5).

L'ordination a permis de confirmer la classification hiérarchique ascendante en les divisant en deux grands axes. L'axe 1 
représente le gradient majeur de l'ordination des relevés qui sépare très nettement les forêts anciennes et les jachères. Les jeunes jachères (1 à 10 ans) se séparent elles aussi très nettement des autres groupes de jachères de plus de 10 ans (Figure 6). Sur l'axe 2, les relevés s'ordonnent à l'intérieur des quatre groupes discriminés par l'axe 1 . Cet axe 2 sépare les relevés effectués au niveau «des forêts anciennes » ou forêts primaires (partie gauche de l'axe 2) des relevés effectués au sein des jachères (partie droite de l'axe 2). La durée de l'abandon de la jachère semble avoir une action amélioratrice de la reconstitution postculturale. Toutefois, pour observer une telle amélioration, ces travaux montrent que la durée des jachères doit être d'au moins 10 ans.

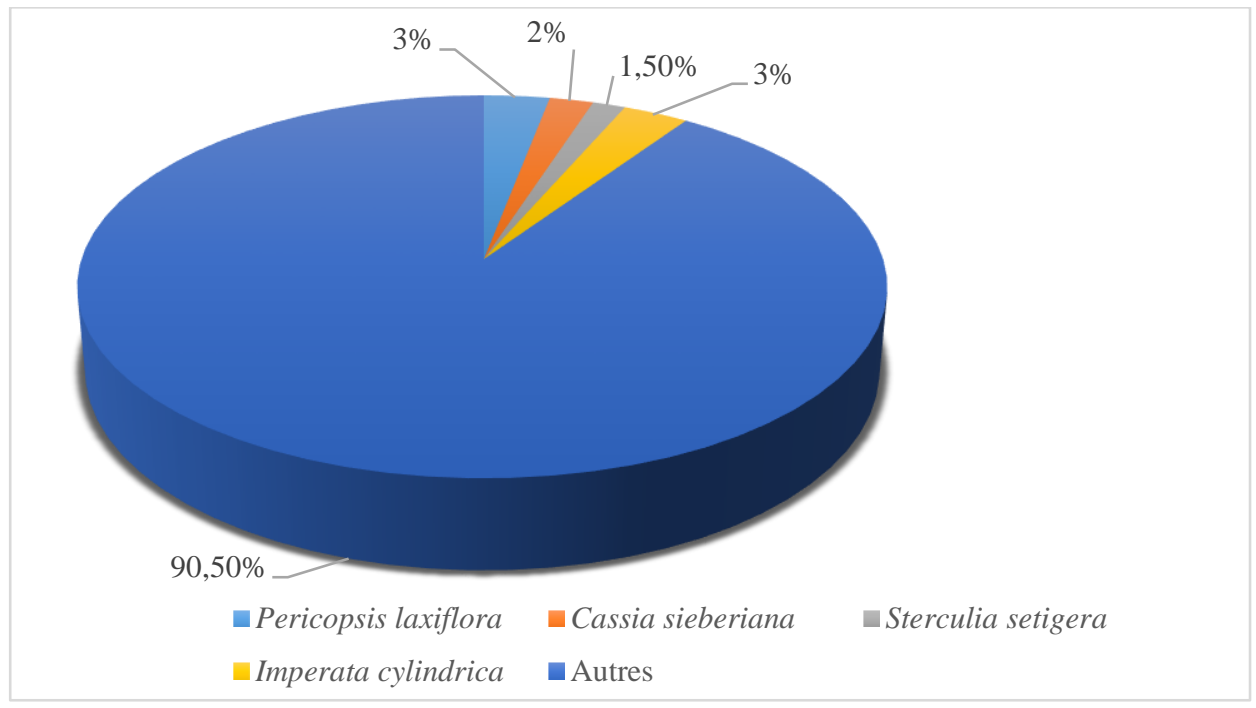

Figure 1 : Spectre des espèces en fonction de leurs nombres d'importances.

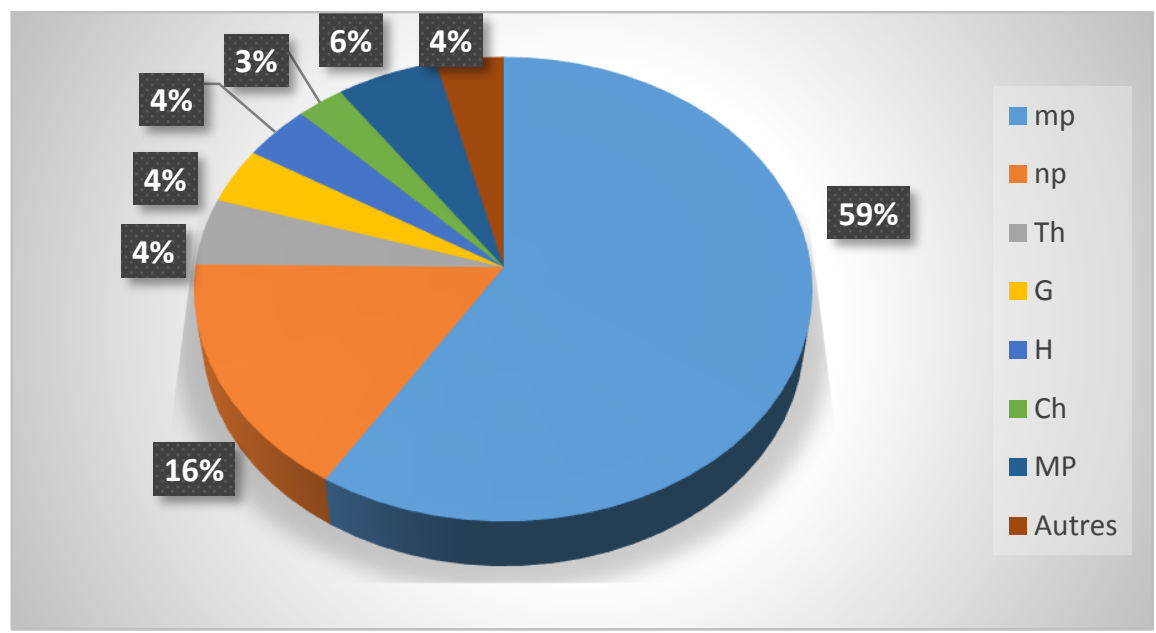

Figure 2 : Spectre du type biologique de la forêt classée de Foumbou.

Légende : Ch : Chaméphytes, G : Geophytes, H : Hémicryptophytes, Hpy : Hydrophytes, mp : Microphanérophytes, np : Nanophytes, Th : Thérophytes. Autres (Mp : Mésophanérophytes, Ept : Epiphytes). 


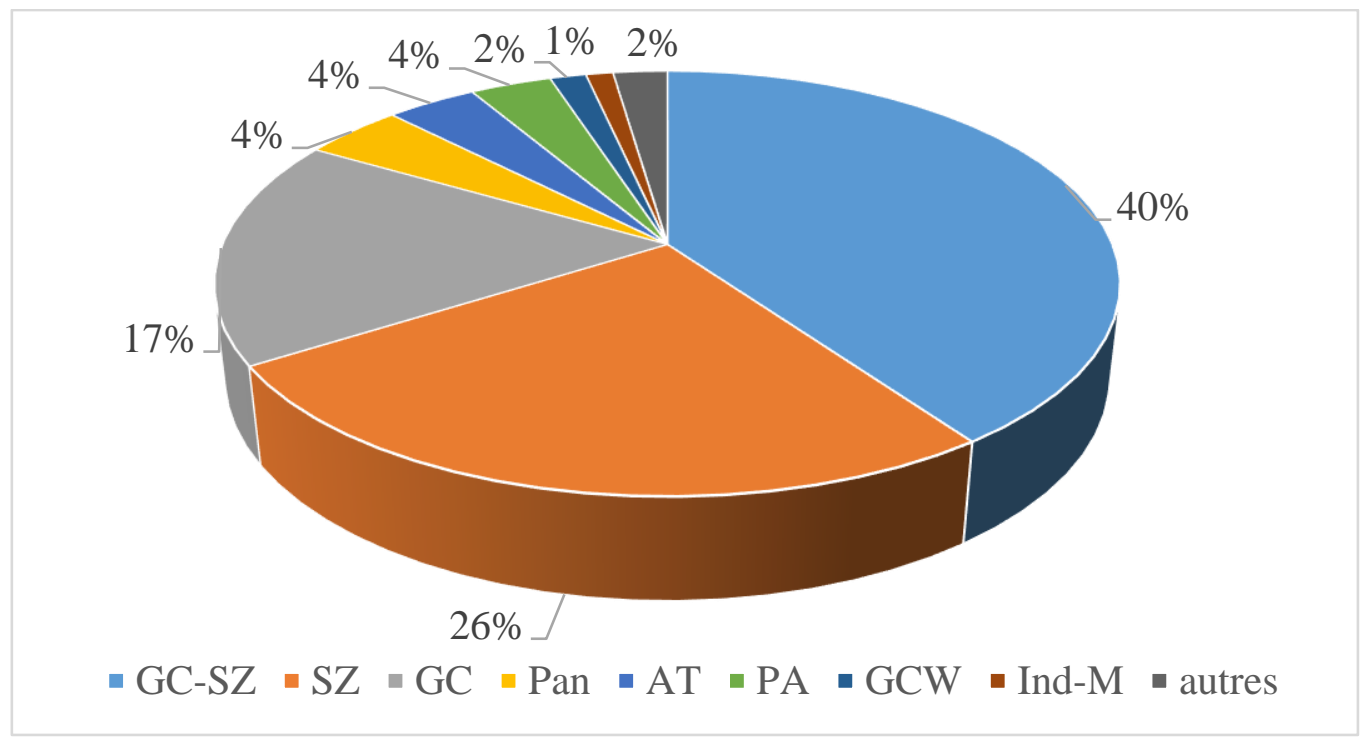

Figure 3 : Spectre du type phytogéographique de la forêt classée de Foumbou.

Légende : AA : Aké-Assi : GC : Taxon de la région Guinéo-congolaise; SZ : Taxon de la région Soudano-Zambésienne; GCSZ: Taxon de la zone de transition Guinéo-congolaise et Soudano-Zambésienne ; Ind-M: Taxon de la région Indo-Maléenne, GCW : Taxon endémique au bloc forestier à l'Ouest du Togo ; Néo: Taxon de la région Néotropicale, PA : plurirégionales africaines.

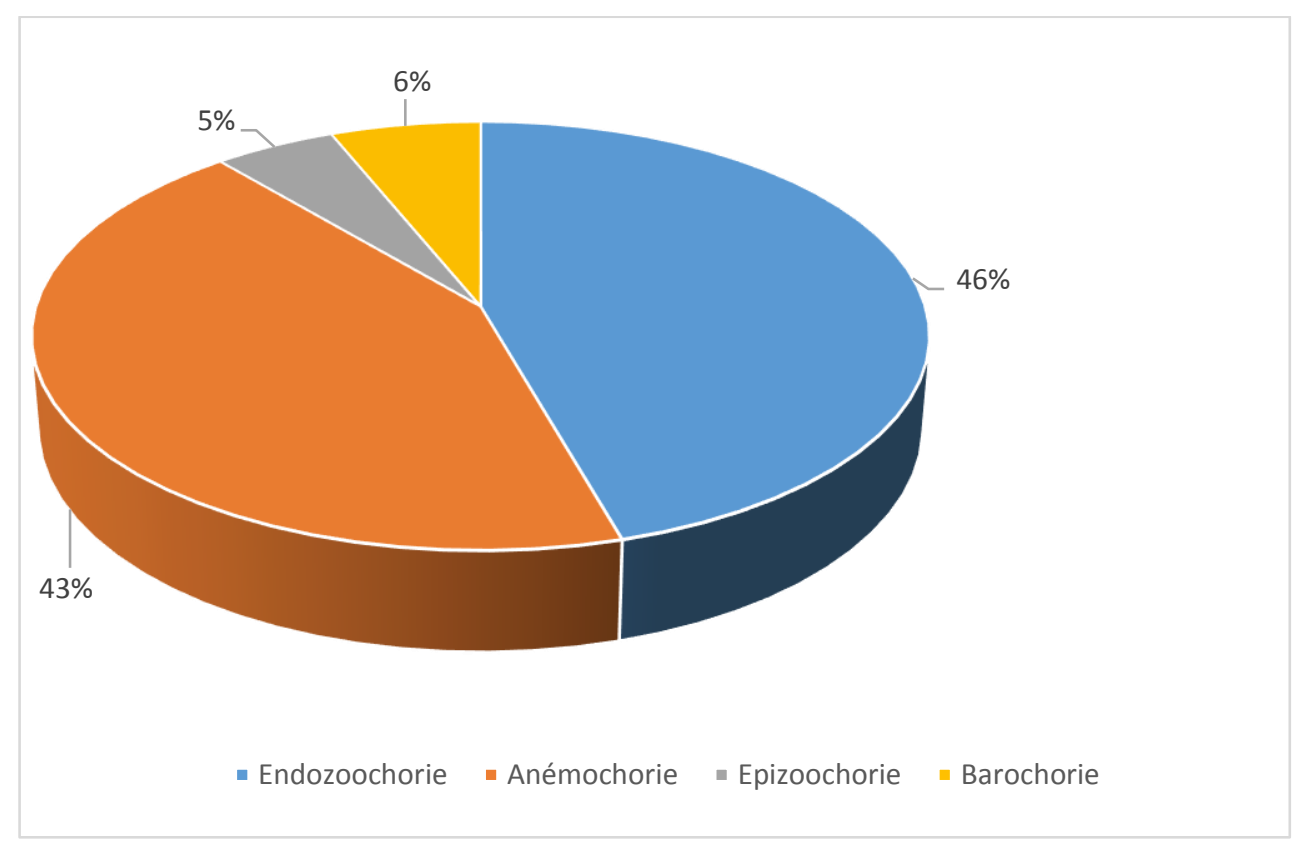

Figure 4 : Spectre du mode de dissémination des diaspores de la forêt classée de Foumbou. 
Tableau 1 : Liste des espèces commerciales et des espèces à statut particulier recensées dans la forêt classée de Foumbou.

\begin{tabular}{|c|c|c|c|c|c|}
\hline $\mathbf{N}^{\circ}$ & Espèces & Familles & Endémisme & UICN & $\begin{array}{l}\text { AA / } \\
\text { Bois d'œuvre }\end{array}$ \\
\hline 1 & Albizia ferruginea (Guill. \& Perr.) Benth. & Fabaceae & & VU & $\mathrm{P} 1$ \\
\hline 2 & Albizia zygia (DC.) J.F. MacBr. & Fabaceae & & & $\mathrm{P} 3$ \\
\hline 3 & Amorphophallus accrensis N. E. Br. & Araceae & GCW & & \\
\hline 4 & Amphimas pterocarpoides Harms & Fabaceae & & $\mathrm{LC}$ & P3 \\
\hline 5 & $\begin{array}{l}\text { Bulbophyllum calyptratum var. lucifugum } \\
\text { (Sum.) Vern. }\end{array}$ & Orchidaceae & GCW & & \\
\hline 6 & Commelina benghalensis L. var. benghalensis & Commelinacea & & $\mathrm{LC}$ & \\
\hline 7 & $\begin{array}{l}\text { Desmodium adscendens (Sw.) DC. var. } \\
\text { adscendens }\end{array}$ & Fabaceae & & $\mathrm{LC}$ & \\
\hline 8 & Desmodium salicifolium (Poir.) DC. & Fabaceae & & $\mathrm{LC}$ & \\
\hline 9 & Detarium senegalense J.F. Gmel. & Fabaceae & & $\mathrm{LC}$ & P3 \\
\hline 10 & Khaya senegalensis (Desv.) A. Juss. & Meliaceae & & & $\mathrm{P} 1$ \\
\hline
\end{tabular}

Légende : UICN : VU -espèce vulnérable ; LC- préoccupation mineure ; LR/LC - risque faible ; LR - donnée insuffisante. AA- plantes rares, devenues rares et en voie d'extinction ou ne se rencontrant qu'exceptionnellement selon Aké-Assi ; Guinéeocongolaises endémiques ouest-africaines ; GCi : Guinéno-congolaises endémiques de Côte d'Ivoire.

Tableau 2 : Indices de diversité des biotopes étudiés.

\begin{tabular}{lll}
\hline Formations végétales & Indices de Shanon & Indice d'équitabilité de Piélou \\
\hline Forêt ancienne & 3,31 & 0,72 \\
Jachère [30ans -35 ans] & 3,29 & 0,78 \\
Jachères [20 ans-30 ans [ & 3,04 & 0,77 \\
Jachères [10 ans-20 ans [ & 3,04 & 0,77 \\
Jachère [1-10 ans] & 2,32 & 0,68 \\
\hline
\end{tabular}

Tableau 3 : Ressemblance floristique des différents biotopes.

\begin{tabular}{llllll}
\hline Biotopes & $\begin{array}{l}\text { Forêt } \\
\text { ancienne }\end{array}$ & $\begin{array}{l}\text { Jachère } \\
\mathbf{3 5} \text { ans }]\end{array}$ & {$\left[\begin{array}{l}\text { Jachère } \\
\mathbf{3 0} \text { ans }]\end{array}\right.$} & $\left.\begin{array}{l}\text { Jachère } \\
\text { 20ans }\end{array}\right]$ & $\begin{array}{l}\text { J11- } \\
\mathbf{1 0} \text { ans }\end{array}$ \\
\hline Forêt ancienne & & & & \\
Jachère $[30-35$ ans $]$ & 0,79 & & & \\
Jachère $[20-30$ ans $]$ & 0,78 & 0,64 & & \\
Jachère [11-20ans] & 0,78 & 0,76 & 0,79 & \\
Jachère $\leq 10$ ans & 0,65 & 0,55 & 0,59 & 0,60 & \\
\hline
\end{tabular}




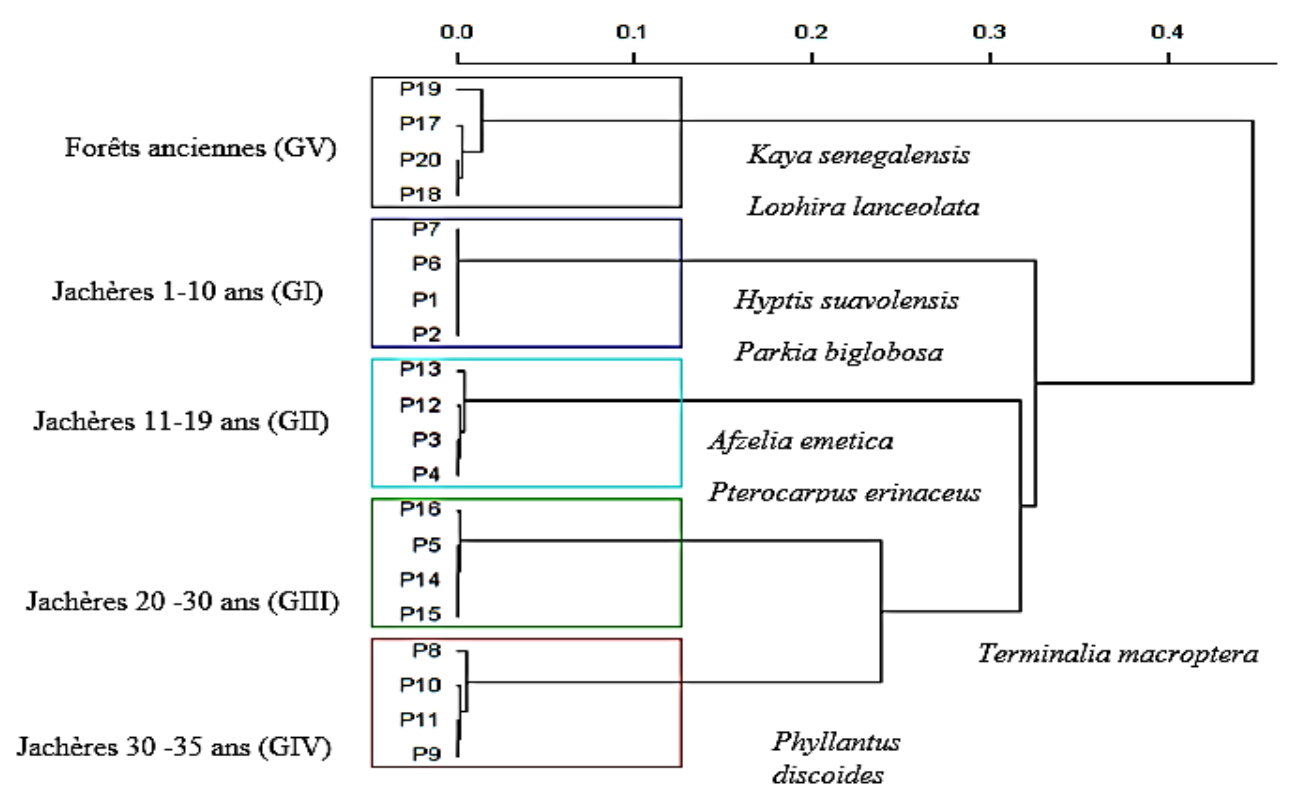

Figure 5: Dendrogramme des affinités floristiques des 20 parcelles inventoriées dans la forêt classée de Foumbou avec les espèces indicatrices.

\section{Factor map}

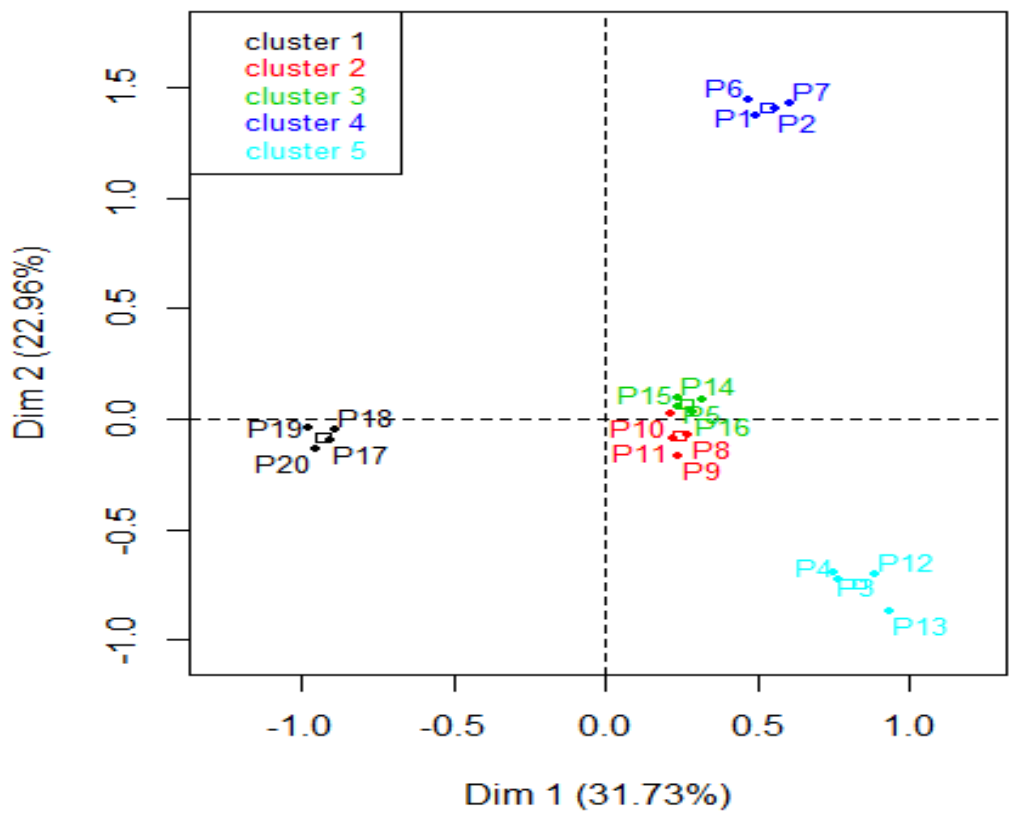

Figure 6: Ordination des 20 relevés de la forêt classée de Foumbou selon l'axe 1 et 2 de la DCA illustrant la disposition des relevés par groupe. 


\section{DISCUSSION}

Ces inventaires botaniques ont permis de dénombrer 259 espèces de plantes vasculaires réparties au sein de 56 familles et 191 genres. Cette diversité est appréciable pour une superficie de 4 ha inventoriées. La faible proportion de la richesse floristique de la forêt classée de Foumbou est due à l'association de biotopes naturels et anthropisés qu'on y trouve. Ces résultats sont similaires à ceux obtenus par N'Guessan (2016) et Sanogo (2020). Ces auteurs, dans leurs travaux respectivement dans la forêt classée de Badénou et dans une forêt privée à Ouahiré (Département de Dianra) dans le Nord de la Côte d'Ivoire, ont obtenu respectivement 241 espèces, 184 genres, 53 familles et 260 espèces, 207 genres et 66 familles. Cette valeur est inférieure à celles obtenues par Gbozé et al. (2020) dans la zone soudanienne en Côte d' Ivoire (350 espèces). La pauvreté relative de cette formation pourrait être principalement liée à l'agriculture et à l'action des feux de brousse qui provoquent une forte perturbation en éliminant certaines espèces avec la création déséquilibre de la végétation. En effet, une fréquence élevée des perturbations limiterait la diversité, seuls quelques taxons parviennent à s'installer et à se maintenir. Pour Gbozé et al. (2020), les plus grandes diversités floristiques sont rattachées à la diversité des habitats ou à l'hétérogénéité environnementale. Les familles botaniques les plus dominantes sur le site sont celles des Fabaceae, des Rubiaceae, des Combretaceae et des Malvaceae. La prédominance de ces familles n'est pas une particularité du site mais une caractéristique générale des forêts qui sont sous la pression anthropiques. Selon Boukpessou (2013), le système de culture itinérant influence la dynamique de peuplement des espèces ligneuses. Cependant, une faible représentation des épiphytes et des Ptéridophytes ont été rencontrées sur le site comme dans les travaux de N'Guessan (2016). Le faible taux d'espèces épiphytes dans la forêt est un caractère normal à cause de la faiblesse de l'humidité du milieu
(N'Da et al., 2008). Le domaine est dominé par les microphanérophytes $(50 \%)$ et les nanophanérophytes (15\%). Mais pris séparément dans les différents biotopes, il existe une dominance parfaite des microphanérophytes. Ce qui traduit une abondance des phanérophytes. Ce constat a été faite par N'Guessan (2016) dans une forêt privée d'Ouahiéré dans le Nord-Ouest de la Côte d'Ivoire. En effet, l'abondance et la dominance des phanérophytes montrent la forte représentativité des ligneux dans presque toutes les formations végétales comme le soutien Issifou et al. (2017) dans ses travaux le démontre également. Ces résultats sont conformes à ceux obtenus par Adjakpa et al. (2013) et Gbozé et al. (2020) qui ont obtenu dans les groupements de formations ligneuses et qui stipule que le fort pourcentage de ces phanérophytes dans les formations végétales est dû aux jeunes individus des espèces ligneuses et aussi à des individus adultes de certaines espèces à valeur socio-économique importante comme Vitellaria paradoxa. D'après Issifou et al. (2017), la forte présence des phanérophytes constitue un espoir pour la reconstitution naturelle des formations végétales dégradées. En effet, l'homme dans ses activités culturales, comme l'agroforesterie opère une sélection qui favorise certaines espèces végétales, influençant ainsi la physionomie de la végétation originelle Gbozé et al. (2020). De la jachère récente à la vieille jachère, il ressort une augmentation de la richesse floristique. L'augmentation de cette richesse floristique pourrait s'expliquer par l'apparition de nouvelles espèces à chaque fois que l'âge de la jachère augmente. En effet, les différents écosystèmes présentent des tailles et des niveaux de diversités spécifiques très différents et en fonction de leurs niveaux de dégradation (Cissé et al., 2020). La végétation rencontrée dans les jachères de 20 ans et plus sont similaires proches mais différentes des forêts anciennes. Ce constat a été également fait par Kassi et Decocq (2006) dans la forêt classée de Sanaimbo. Ces nouvelles espèces 
sont soit introduites par les oiseaux migrateurs, les animaux ou le vent soit issues des rejets. L'apparition de ces nouvelles espèces pourrait encourager la SODEFOR à promouvoir une bonne gestion participative dans le but de favoriser une bonne résilience. La plus faible richesse floristique enregistrée dans les jeunes jachères, expliquent mieux les fortes dégradations observées dans la zone d'étude du fait de l'influence humaine. Adjakpa et al. (2013) parviennent à la même conclusion dans le Bassin Moyen de la Sota au Nord-Bénin. Les valeurs relativement élevées de l'indice d'équitabilité de Pielou $(0,68$ à 0,78$)$ concordent avec les observations faites par Gbozé et al. (2020). Pour ces auteurs, il n'y a pas d'espèce dominante dans les jachères postculturales en zone de savane soudanienne comme c'est le cas en zone de forêt dense dans les travaux de N'Guessan (2018) dans la forêt classée d'Agbo I. La forêt de Foumbou renferme deux espèces devenues rares selon Aké-Assi (2002). Il s'agit de Detarium microcarpum et Syzygium guineense. Elle regorge aussi des espèces qui figurent sur la liste de l'UICN (2020). Elle compte deux espèces endémiques Ouest africaines (Amorphophallus accrensi, Bulbophyllum calyptratum). Les espèces à statut particulier dans les biotopes inventoriés présentent une faible richesse. Leur faible présence indique que la végétation est fortement perturbée à l'issue des activités anthropiques (Abrou et al., 2019). Alors, la petite proportion de ces espèces à valeur de conservation confirme également que ces formations végétales de la zone d'étude sont des écosystèmes fragiles et qu'il faut nécessairement les préservés. C'est dans cette même logique que Myers et al. (2000) soulignent que les espèces endémiques, rares et menacées d'extinction sont celles pour lesquelles les efforts de conservation doivent être prioritaires. Ce présent travail montre une prédominance des espèces endémiques de l'aire de transition GC-SZ et des espèces soudano-zambéziennes, confirmant la mosaïque forestière et savanicole attribuée à la forêt classée et à la zone de forêt claire en Côte d'Ivoire (Kassi, 2006). La forte présence des espèces de la région Guinéo-Congolaise (GC) est une preuve que cette zone appartient à la zone de transition Guinéo-Congolaise (Tiokeng, 2015). Plusieurs auteurs (Tiebré et al., 2016 ; Sanogo, 2020) ont fait les mêmes constats en ayant étudié la reconstitution des jachères post culturales. Tous ces auteurs sont unanimes que ces caractéristiques constituent des bons outils d'appréciation de la maturité des formations végétales. La forte proportion des espèces de base-soudanienne combinée à celle des espèces soudano-zambéziennes et soudano-guinéenne traduit l'appartenance de la zone d'étude au domaine soudanien (Adomou, 2005). Les résultats observés montrent que la végétation des jachères les plus âgées (30 ans et plus) sont relativement proche de celle rencontrée dans les forêts anciennes. Ceci suggère que la forêt de Foumbou présenterait une bonne résilience face aux perturbations anthropiques que sont les feux de brousse, l'agriculture et l'orpaillage clandestin. De plus, l'intervention humaine, se traduisant par des coupes de bois, de carbonisation et de défrichements, contribue fortement à la dégradation des différentes formations végétales. Une telle évolution d'anthropisation conduit à court et à moyen terme à une réduction de la production de biens et de services et à long terme au point de basculement. D'après Adjakpa et al. (2013), lorsqu'un écosystème perd sa capacité de récupération, elle perd également sa capacité de résilience. Le temps de repos qui suit la culture se caractérise par une dynamique progressive de la végétation (Adingra, 2017). La classification confirme que la reconstitution des jachères post-culturales ou écosystèmes culturaux «abandonnés » subie une dynamique progressive de la végétation. Le processus de recolonisation de la jachère s'effectue suivant quatre étapes successives. Cette dynamique évolutive est fonction du temps mais aussi de plusieurs facteurs environnementaux. La classification hiérarchique montre que la 
composition floristique des relevés effectués dans la forêt de Foumbou est étroitement dépendante de l'âge des jachères où ils ont été relevés. Ces résultats confirment ceux de Kassi et Decocq (2006) qui expliquent que le dynamisme de la végétation est fonction de l'évolution floristique et structurelle. Ces résultats mettent également en évidence un lien étroit entre le mode de dispersion des espèces et le niveau de perturbation des différents biotopes. Ainsi, pour Kassi (2006), les jeunes jachères sont riches en espèces pionnières héliophiles. C'est aussi le cas dans ces travaux où les premiers stades de la succession sont caractérisés par des espèces savanicoles plus favorables à un grand ensoleillement. Les espèces de forêts claires, elles au contraire caractérisent les jachères les plus âgées où les conditions de leur mise en place sont réunies. Dans la zone d'étude, comme dans les travaux de Gbozé et al. (2020), les perturbations sont généralement dues aux feux de brousses, aux facteurs climatiques tels que la sécheresse, le décalage des saisons et les facteurs édaphiques, etc. Cependant, la présence d'une espèce dans un milieu post-cultural est fonction de l'activité exercée dans le milieu. Ce qui explique la présence de certaines espèces comme Parkia biglobosa dans les jeunes jachères et témoigne de la pratique de système d'agroforesterie par les paysans. La présence de grands individus de cette espèce dans les jeunes jachères est simplement due au fait que les individus de cette espèce sont épargnés par les agriculteurs lors de la création des champs. Pour Gbozé et al. (2020), le déroulement des successions se repose sur l'instauration d'une catégorisation des végétaux en fonction de leurs capacités d'exploitation des ressources abiotiques. Pour ces auteurs, les changements de végétation durant la succession secondaire sont principalement dus aux interactions biotiques c'est-à-dire aux capacités des plantes à modifier leur environnement de manière à faciliter leur maintien, l'installation d'autres espèces et/ou à favoriser des interactions compétitives et la tolérance des autres espèces.
De même, Kassi et Decocq (2006), se sont intéressés à l'étude des espèces pionnières à croissance rapide et ont conclu que certaines espèces disparaissent rapidement, lorsque la végétation acquiert progressivement une physionomie forestière et que les strates supérieures les privent de lumières. $\mathrm{Ce}$ renouvellement d'espèces ne semble donc pas durablement altérer le fonctionnement de l'écosystème. Au contraire, il y contribue au bon déroulement de la succession secondaire.

L'ordination montre que le principal gradient responsable de la structuration des communautés végétales est un gradient de maturité, qui se traduit de manière complexe sur les facteurs environnementaux. La végétation des jachères les plus âgées (âge supérieur à 20 ans) est relativement proche de celle rencontrée en système de forêt ancienne. Ceci suggère que la forêt de Foumbou présenterait une bonne résilience face aux perturbations, bien que les vieilles jachères demeurent moins diversifiées que la forêt ancienne (forêt claire). Ces observations sont similaires à celles des travaux de Tiebré et al. (2016) dans la zone de transition forêt - savane. La dispersion des relevés dans le plan factoriel suggère que les stades initiaux de la recolonisation sont aléatoires. Ces jeunes stades seraient menacés par l'effet des pâturages, l'exploitation clandestine, des feux de brousse. Ces résultats confortent l'hypothèse de N'Guessan (2018) qui dit que, bien que l'âge des jachères soit le principal facteur expliquant la structuration des communautés végétales et leur composition floristique, plusieurs autres facteurs sont également susceptibles d'intervenir.

\section{Conclusion}

Cette étude a permis de déterminer les différents types de végétations rencontrées dans la forêt classée de Foumbou. . Ces biotopes sont importants car ils renferment de nombreuses espèces forestières. Les inventaires botaniques réalisés montrent l'importance floristique de cet écosystème 
forestier pour la région de Korhogo. Ainsi, ces travaux montrent que la flore étudiée est diversifiée. Elle renferme 259 espèces, réparties en 191 genres et 56 familles. Au niveau des familles recensées, sept (7) d'entre elles, notamment les Fabaceae, les Rubiaceae, les Combretaceae, les Malvaceae, les Moraceae, les Poaceae et les Lamiaceae, sont les plus fournies et renferment $51,56 \%$ de l'ensemble des espèces inventoriées. L'analyse des types biologiques révèle que les Phanérophytes sont les plus observés. Pour ce qui est des modes de dissémination des diaspores, les espèces endozoochores (46\%) et anémochores (43\%) sont les plus fournies. La richesse de la flore de cette aire protégée est aussi caractérisée par la présence de deux espèces devenues rares selon Aké-Assi : Detarium microcarpum et Syzygium guineense, la présence des espèces de la liste rouge de l'UICN et des espèces endémiques Ouest africaines (Amorphophallus accrensi, Bulbophyllum calyptratum). Cela donne un intérêt particulier pour la conservation de la forêt classée de Foumbou. Malheureusement, la forêt classée de Foumbou subit encore d'énormes pressions anthropiques. Les efforts de conservation des espèces endémiques, des espèces rares et des espèces menacées d'extinction doivent être une priorité. Des zones de frontières floues et d'âges différents, témoignent des étapes de la régénération de la végétation. Ainsi, dès l'abandon des champs, on assiste à une dynamique progressive de la végétation, on passe alors progressivement des jeunes jachères à des vieilles jachères. Le nombre d'espèces présentes dans les différentes classes d'âges serait fonction de la stabilité du milieu. Malgré les menaces que subissent sur cette forêt classée, ces travaux montrent que la forêt de Foumbou regorge encore des zones de forêts claires ou forêt ancienne. Face à l'intensification du phénomène $\mathrm{du}$ changement climatique entrainant des conséquences grandissantes, la conservation des forêts classées constitue toujours une contribution potentielle à la résolution du problème.

\section{CONFLIT D'INTERETS}

Les auteurs de cet article déclarent qu'il n'y a aucun conflit d'intérêts.

\section{CONTRIBUTIONS DES AUTEURS}

AT a contribué à la collecte des données, à la conception et à la structuration de l'étude, à la recherche documentaire et à la rédaction de l'article. AEN a participé à la conception du manuscrit. JNK a participé à la correction du manuscrit.

\section{REMERCIEMENTS}

Ces travaux ont été réalisés avec le soutien du MESRS de Côte d'Ivoire dans le cadre de la mise en œuvre du C2D par l'IRD et facilité par le projet DynRecSE, du programme PReSeD-CI 2. Nous remercions la population riveraine de la forêt classée de Foumbou.

\section{RÉFÉRENCES}

Abrou NEJ, Kouamé D, Adou Yao CY. 2019. Diversité floristique des communautés végétales dans l'espace de la Forêt des Marais Tanoé-Ehy (FMTE), Sud-est de la Côte d'Ivoire. International Journal of Biological and Chemical Sciences, 13(6): 2874-2887.

DOI: https://dx.doi.org/10.4314/ijbcs.v13i6.35

Adingra MMAO. 2017. Dynamique du peuplement et stocks de carbone dans la mosaïque de végétation de la forêt classée de Bamo (Côte d'Ivoire). Thèse Doctorat, Université Félix HouphouëtBoigny, Côte d'Ivoire, $158 \mathrm{p}$.

Adjakpa JB, Yédomonhan H, Ahoton L, Weesie P, Akpo L. 2013. Structure et diversité floristique des îlots de forêts riveraines communautaires de la Basse vallée de la Sô au Sud-Est du Bénin. Journal of Applied Biosciences, 65: 4902 - 4911. DOI:10.4314/JAB.V65I0.89573 
Adjanohoun E. 1964.Végétation des Savanes et des Rochers Découverts en Côte d'Ivoire. ORSTOM: Paris.

Adomou AC. 2005. Vegetation patterns and environmental gradients in Benin: Implications of biogeography and conservation. Ph.D Thesis, Wageningen University, Wageningen, $150 \mathrm{p}$.

Aké-Assi L. 1984. Flore de la Côte d'Ivoire : Etude descriptive et biogéographique avec quelques notes ethnobotaniques. Thèse Doctorat d'Etat, Université Nationale d'Abidjan, Côte d'Ivoire, 1206 p.

Aké-Assi L. 2001- 2022. Flore de Côte d'Ivoire 1: Catalogue Systématique, Biogéographie et Ecologie. Conservatoire et Jardin Botaniques: Genève (Suisse).

Akoegninou A. 2006. Flore Analytique du Bénin. Backhuys Publishers: Wageningen.

Akossongo TJ. 2004. Rapport national sur la gestion durable des forêts au BurkinaFaso. Rapport MECV/BF, Burkina-Faso, $100 \mathrm{p}$.

Amon A. 1995. Contribution à l'aménagement de la forêt classée (plan de gestion des reboisements). Mémoire de DEA, INPH, Côte d'Ivoire, $54 \mathrm{p}$.

APG IV. 2016. An update of the Angiosperm Phylogeny Group classification for the ordersand families of flowering plants. Botanical Journal of the Linnean Society, 181: 1-20. DOI: https://doi.org/10.1111/boj.12385

Arbonnier M. 2002. Arbres, Arbustes et Lianes des Zones Sèches de l'Afrique de l'Ouest. Edition CIRAD-MNHN : Paris.

Bayen P. 2016. Restauration des sols dégradés par afforestation et évaluation des potentialités de séquestration du carbone de six espèces ligneuses en zone sahélienne et soudano-sahélienne du Burkina Faso. Thèse de Doctorat, Université Ouaga 1 Pr Joseph KIZERBO, Burkina Faso, 121p.
Blondel J. 1979. Biologie et Ecologie. Masson \& Cie : Paris.

Cissé A, Ouattara M, N'guessan EA, Abrou JEN. 2020. Diversité végétale et usages des plantes dans une zone de savane soudanienne : Cas de la localité de Ferkessédougou (Nord, Côte d'Ivoire). Int. J. Biol. Chem. Sci., 14(8): 2807-2825. DOI: $10.4314 /$ ijbcs.v14i8.13

FAO. 2002. Evaluation des ressources forestières mondiales 2000. Rapport principal. FAO, Rome-Italie.

FAO. 2015. Evaluation des ressources forestières mondiales: Comment les forêts sont gérées dans le monde. Répertoire de données de la FAO, RomeItalie,

$23 \mathrm{p}$. https://www.fao.org/3/i4808f/i4808f.pdf.

FAO. 2020. Evaluation des ressources forestières mondiales 2020 - Principaux résultats. FAO, Rome. DOI: https:// doi.org/10.4060/ca8753fr.

Fearnside P. 2005. Deforestation in Brazilian Amazonia: history, rates and consequences. Conservation Biology, 19(3): 680-688. DOI: $10.1111 / \mathrm{j} .1523-$ 1739.2005.00697.x

Gbozé AE, Adingra OMM, Sanogo A, Kassi N'J. 2020. Systematic and plant geography analysis of Badénou forest (Korhogo, Côte d'Ivoire). Int. J. Biol. Chem. Sci., 14(9): 3156-3167. DOI: 10.4314/ijbcs.v14i9.15

Guillaumet JL, Adjanohoun E. 1971. La végétation de la Côte d'Ivoire. In Le Milieu Naturel de la Côte d'Ivoire, Avenard JM, Eldin E, Girard G, Sircoulon J, Touchebeuf P, Guillaumet JL, Adjanohoun E, Perraud A (eds). ORSTOM : Paris ; 157-263.

Hawthorne WD. 1996. Guide de terrain pour les arbres des forêts denses de la Côte d'Ivoire et des pays limitrophes avec clés végétatifs sur plus de 650 espèces d'arbres, à partir de $5 \mathrm{~cm}$ de diamètre. Thèse, Université d'Agronomique de Wageningen, Pays Bas, 279 p. 
Issifou MY, Arouna O, Zakari S. 2017. Diversité floristique et structure des formations végétales dans le district phytogéographique du Borgou-nord au Benin (secteur de l'arrondissement de bagou). Thèse, Université de Lomé, Togo, 63-80.

Kassi NJ. 2006. Successions secondaires postculturales en forêt dense semi-décidue (Côte d'Ivoire): nature, structure et organisation fonctionnelle de la végétation. Thèse Doctorat, Université de Picardie Jules Verne d'Amiens, France, $212 \mathrm{p}$

Kassi NJ, Decocq G. 2006. Régénération de la forêt dense semi-décidue dans les stades post-culturaux en forêt classée de Sanaimbo (Côte d'Ivoire). Acta Botanica Gallica, 154(3): 395-405. DOI: 10.1080/12538078.2007.10516072

Kassi NJ, Yongo DO, Decocq G. 2011. L'intérêt d'une approche systémique des dynamiques de végétations en phytosociologie forestière tropicale : cas de la forêt classée de Sanaimbo à Bongouanou/Dimbokro (Côte d'Ivoire). Int. J. Biol. Chem. Sci., 5(3): 1337-1350. DOI: 10.4314/ijbcs.v5i3.72288

Kouadio YJC, Soiret kS, Kpan WB, Yao NO, N'guessan KE, Kouassi KP, Gomez PJ. 2018. Valeur de conservation de la Réserve Naturelle Volontaire (RNV) de la Dodo, Sud-Ouest de la Côte d'Ivoire (Afrique de l'Ouest). Int. J. Biol. Chem. Sci., 12(6): 2784-2796. DOI: 10.4314/ijbcs.v12i6.24

Kouamé NF. 1998.-Influence de l'exploitation forestière sur la végétation et la flore de la forêt classée du Haut-Sassandra (CentreOuest de la Côte d'Ivoire). Thèse Doctorat $3^{\text {e }}$ Cycle, UFR Biosciences, Université Cocody-Abidjan, Côte d'Ivoire, $227 \mathrm{p}$.

Lebrun J. 1947. Végétation de la Plaine Alluviale au Sud du Lac Edouard (ed). Expl. Parc National Albert : Bruxelles.
Lebrun JP, Stork AL. 1991-1997. Enumération des plantes à Fleurs d'Afrique Tropicale. Conservatoire et Jardin Botaniques de la Ville de Genève : Genève.

Locatelli B, Loisel C. 2002. Changement climatique : la vérité est-elle au fond du puits ? Une analyse des controverses sur les puits de carbone. Natures Sciences Sociétés, 10(4): 7-19. DOI: https://doi.org/10.1051/nss/20021004007

Magurran AE. 2004. Measursuring Biological Diversity. Blackwelll Publ.: Malden.

McCune B, Mefford MJ. 2002. PC-Ord, Multivariate Analysis of Ecological Data (version 4). MjM Software Design, Gleneden Beach.

Martel S, picard O. 2013. Empreinte carbone territoriale forestière : exemple des pays de la Vallée de la Sarthe et des Mauges. CNPF, 57-59.

N'Da DH, Adou YCY, N'Guessan KE, Koné M, Sangne YC. 2008. Analyse de la diversité floristique du Parc National de la Marahoué, Centre-ouest de la Côte d'Ivoire. Afrique Science, 4(3): 552-579. DOI: $10.4314 /$ afsci.v4i3.61700

Marion J. 2018. Le rôle de la diversité sur la stabilité des processus des écosystèmes forestiers en contexte de changement climatique. Thèse de Doctorat, AgroParis-Tech, Université de Montpellier, France, $361 \mathrm{p}$.

Myers N, Mittermeier RA, Mittermeier CG, Da Fonseca GA, Kent J. 2000. Biodiversity hotspots for conservation priorities. Nature, 403(6772): $853 . \quad$ DOI: $10.1038 / 35051132$

N'Guessan AE. 2016. Diversité floristique d'une forêt privée à Dianra au Nord de la Côte d'Ivoire. Master, UFR Biosciences, Université Félix Houphouët-Boigny, 51 p.

N'Guessan AE. 2018. Dynamique de la végétation et facteurs de reconstitution de la biomasse des forêts secondaires dans la forêt classée d'Agbo 1 (Côte d'Ivoire). 
Thèse Doctorat, Université Félix Houphouët- Boigny, Côte d'Ivoire, 179 p.

N'Guessan AE, Kassi N'J, Yao NO, Amani HKB, Gouli GZR, Piponiot C, Irie Bi CZ, Hérault B. 2019. Drivers of biomass recovery in a secondary forested landscape of West Africa. Forest Ecology and Management, 433: 325 - 331. DOI: 10.1016/j.foreco.2018.11.021

N'Guessan KE. 2011. Etude d'impact environnemental et social du projet d'exploitation de la mine de manganèse de Ziemougoula (Odienné). Rapport d'études floristiques, Ziemougoula, 65 p.

Pielou EC. 1966. The measurement of diversity in different types of biological collections. J. Theor. Biol., 13: 131-144. DOI: https://doi.org/10.1016/00225193(66)90013-0

Rarivoson NTJ. 2019. Quantification de la biomasse aérienne et évaluation du stock de carbone par la méthode de regression allométrique: cas de la forêt haute littorale de Tampalo (Madagascar). Mémoire de Licence, Université d'Antananarivo, Antananarivo, $59 \mathrm{p}$.

Raunkiaer C. 1934. The Lifes Forms of plants and Statistical Plant Geography. Oxford University Press : London.

Roxane S. 2015. Evaluation de la capacité adaptative des socio-écosystèmes forestiers français face au changement climatique : le cas de la migration assistée. Mémoire de Biodiversité et Ecologie, Université Paris Saclay (COmUE), $56 \mathrm{p}$.

Saïdou A, Dossa AFE, Gnanglè PC, Balogoun I, Aho N. 2012. Evaluation du stock de carbone dans les systèmes agroforestiers à karité (Vitellaria paradoxa C.F. Gaertn.) et à néré (Parkia biglobosa Jacq. G. Don) en zone Soudanienne du Bénin. Bulletin de la Recherche Agronomique du Bénin (BRAB), Bénin, 9 p. http://www.slire.net/document/1737

Sanogo A. 2020. Diversité floristique et valeur de conservation de la forêt classée de
Badénou (Korhogo, Côte d'Ivoire). Mémoire de Master, Université Félix Houphouët-Boigny, Côte d'Ivoire, 53 p.

Senterre B. $2005 . \quad$ Recherches méthodologiques pour la typologie de la végétation et la phytogéographie des forêts denses d'Afrique tropicale. Thèse Doctorat, Université Libre de Bruxelles, Bruxelles, $345 \mathrm{p}$.

Shannon CE. 1948. A mathematical theory of communication. Bell System Technical Journal, 27: 379-423. DOI: https://doi.org/10.1002/j.15387305.1948.tb01338.x

Tiebré MS, Ouattara D, Kpangui KB, Kouassi DF, N'Guessan KE. 2016. Diversité floristique de la région de Foungbesso en zone de transition forêt - savane à l'ouest de la Côte d'Ivoire. Int. J. Biol. Chem. Sci., 10(3): 1007-1016. DOI: 10.4314/ijbcs.v10i3.8

Tiokeng B, Mapongmetsem P, Nguetsop VF, Tacham WN. 2015. Biodiversité floristique et régénération naturelle sur les Hautes Terre de Lebialem (Ouest Cameroun). International Journal of Biological and Chemical Sciences, 9(1): 56-68

DOI: http://dx.doi.org/10.4314/ijbcs.v9i1.6

Toko MI, Touré F, Toko II, Sinsin B. 2012. Indices de structures spatiales des îlots de forêts denses dans la région des Monts Kouffé. Vertigo-la Revue Electronique en Sciences de l'Environnement, 12(3). DOI: 10.4000/vertigo. 13059

UICN (International Union for Concervation of Nature). 2020. UICN Red List of Threatened Species. UICN, Version 2020.2. www.iucnredlist.org

White F. 1983.The vegetation of Africa, a descriptive memoir to accompany the UNESCO-AETFA-UNSO vegetation map of Africa. UNESCO, Paris.

White F. 1986. La Végétation de l'Afrique. Jard. Nat. : Belgique. 